\title{
Die Fragmente-Sammlung Ms. 375 der Universitätsbibliothek Marburg
}

\author{
Entstehung, Stand und Perspektiven
}

\author{
Diana Müller
}

$\mathrm{D}$ ie Universitätsbibliothek der 1527 gegründeten Philipps-Universität Marburg besitzt eine kleine Sammlung von gut einhundert mittelalterlichen Buchhandschriften, die jedoch von einer überraschend großen Zahl von mehreren Hundert Fragmenten flankiert wird. Es handelt sich bei diesen Fragmenten um eine nahezu unerschlossene Bestandsgruppe. Zwar sind die mittelalterlichen Handschriften der Universitätsbibliothek Marburg im Handschriftenkatalog von Sirka Heyne aus dem Jahr 2002 verzeichnet. ${ }^{1}$ Ausdrücklich unberücksichtigt aber blieben bei dem damaligen Katalogisierungsvorhaben - von einigen Ausnahmen abgesehen - die umfangreichen Fragment-Bestände, die gesammelt unter der Signatur Ms. 375 zusammengefasst sind. ${ }^{2}$ Erfasst wurde im Heyne-Katalog neben den 102 Handschriften lediglich eine enge Auswahl von I4 Fragmenten. Ausgewählt wurden dabei hauptsächlich deutschsprachige Fragmente, einige frühe Fragmente sowie Fragmente, die nicht unter Ms. 375, sondern unter einer eigenen Signatur geführt werden. ${ }^{3}$

Die ersten Handschriften - nicht viel mehr als eine Handvoll - kamen bereits kurz nach der Gründung der Philipps-Universität in die Büchersammlung der Universität, als im Zuge der Reformation umliegende hessische Klöster in Alsfeld, Marburg und Haina aufgelöst wurden. ${ }^{4}$ Wohl im mittleren Drittel des 17. Jahrhunderts kam aller Wahrscheinlichkeit nach durch den Schweizer Theologen und Alchemisten Raphael Egli (1559-1622) 5 eine nicht nur medizingeschichtlich herausragende Handschriftengruppe hinzu, die ungefähr ein Viertel des gesamten Handschriften- bestandes ausmacht. Es handelt sich um Bände aus dem Vorbesitz des Züricher Arztes Christoph Clauser, die unter anderem Autographen von Hans Minner (I415-I480) enthalten, ${ }^{6}$ der in der Forschung als bedeutendster deutscher Apotheker des Mittelalters gilt.7 Im Jahr I8ı2 schließlich wurde eine größere Zahl Handschriften aus dem aufgehobenen Kloster Corvey, darunter auch ehemalige Bände der Bursfelder Klosterbibliothek, in den Marburger Bestand aufgenommen. ${ }^{8}$ Diese Handschriften aus Säkularisierungsgut machen ein Drittel des Bestandes aus. Die restlichen Handschriften kamen im 19. und 20. Jahrhundert hinzu. Hierzu gehören etwa Codices, die nach der Schließung der Universität Rinteln nach Marburg gelangten, sowie Handschriften aus Professorennachlässen und aus vereinzelten Erwerbungen, ${ }^{9}$ wie Ms. 657, die von der Universitätsbibliothek im November 1937 auf einer Auktion der Fa. Karl \& Faber in München ersteigert wurde.

Die umfangreichen Fragmentbestände unter Ms. 375, um die es in diesem Beitrag gehen soll, stellen im Wesentlichen Makulaturfragmente dar, die während des 19. und 20. Jahrhunderts aus Drucken ausgelöst und separiert wurden, aber wohl auch aus Käufen und Schenkungen stammen. ${ }^{10}$ Dass Drucke oder auch Buchhandschriften überhaupt regelmäßig auch Träger von Handschriftenfragmenten sind, ist dabei dem Prozess der Buchherstellung geschuldet, denn Handschriftenfragmente fanden bis ins 17. Jahrhundert hinein in Form von Makulaturmaterial als Falzverstärkung oder Einbanddeckeln Verwendung. Auch in Vorder- und Hinterspiegel können häufig Fragmente oder Fragmentteile verborgen sein. ${ }^{\text {II }}$ 
Über die Anfänge der Fragmente-Sammlung der Universitätsbibliothek Marburg gibt es kaum greifbare Informationen. Eine kurze Erwähnung findet die Sammlung bei Dirk Barth, dem damaligen Direktor der Universitätsbibliothek, im Vorwort des Handschriftenkatalogs von Heyne, wo es heißt: „Und was die Fragmente betrifft, ist ohnehin erst nach Hermann damit begonnen worden, diese in größerem Umfang aus Bucheinbänden des Altbestandes herauszulösen und in einer besonderen Sammlung zu vereinigen. " ${ }^{12} \mathrm{Da}$ Carl Friedrich Hermann I842 von der PhilippsUniversität an die Universität Göttingen wechselte, ${ }^{\text {,3 }}$ könnte die Sammlung folglich ab der zweiten Hälfte, wahrscheinlicher aber gegen Ende des I9. Jahrhunderts begonnen worden sein, als dies auch an anderen Bibliotheken üblich wurde. ${ }^{14}$ Fortgeführt wurde diese Praxis in Marburg dann vermutlich bis in die I98oer Jahre. ${ }^{15}$

Heute besteht die Fragmente-Sammlung der Universitätsbibliothek Marburg aus mehreren Hundert Fragmenten, die in Archivkisten lagern (Abb. I). Verteilt auf sechs Kisten finden sich darin insgesamt ca. 550 Blätter, ${ }^{16}$ darunter Einzelblätter, Doppelblätter und Makulaturmaterial. Überwiegend handelt es sich dabei um lateinische, in einigen Fällen aber auch um volkssprachige Fragmente. Die Entstehungszeit der Bruchstücke reicht dabei von der Zeit um 800 bis ins 15 . Jahrhundert.

Zusammengehalten wird die FragmenteSammlung durch eine Gruppensignatur (Ms. 375), die um eine laufende Zählung für die Einzelstücke ergänzt wird. ${ }^{17}$ Die Stückzählung bezieht sich dabei auf die einzelnen Blätter, wobei die einzelnen Signaturen zusammengehörige Fragmentteile berücksichtigen und abbilden, wie das beispielsweise bei Ms. 375/I-3+Ia der Fall ist: Es handelt sich um vier zusammengehörige Fragmentteile derselben Handschrift, die auch zusammen in einer Mappe aufbewahrt werden (Abb. 2).

Wie anfangs erwähnt, wurde in den Heyne$\mathrm{Katalog}^{18}$ lediglich eine kleine Auswahl von Fragmenten aufgenommen und beschrieben. Davon beziehen sich vier Beschreibungen auf Fragmente aus Ms. 375, nämlich Ms. 375/ I-3 (Priscianus Caesariensis, um 8oo), Ms. 375/17-I8 (Cassianus, I. Hälfte 9. Jh.), Ms. 375/28-3I (Breviarium, Anfang II. Jh.) und Ms. 375/85-86 (Bonaventura, I. Hälfte I4. Jh.). Angemerkt sei hier noch, dass der Heyne-Katalog aus dem Jahr 2002 Ms. 375/ra nicht enthält, da dieser Teil einer frühen Priscian-Handschrift aus der Zeit um 800 bis zum Jahr 2004 ungezählt und unbeschrieben bei den anderen Blättern lag. ${ }^{19}$ Die Fragmente Ms. 375/I3 (ebenfalls noch ohne ia), Ms. 375/5-Io, Ms. 375/II, Ms. 375/I2, Ms. 375/13-I6, Ms. 375/17-I8, Ms. 375/19, Ms. 375/24-25, Ms. 375/69-70 und Ms. $375 / 74$ sind darüber hinaus in Bernhard Bischoffs Katalog der festländischen Handschriften des neunten Jahrhunderts aus dem Jahr 2004 verzeichnet. ${ }^{20}$ Es gibt außerdem ein unveröffentlichtes Handschrifteninventar für den Dienstgebrauch an der Universitätsbibliothek Marburg, in dem Kurzbeschreibungen der Fragmente Ms. 375/I bis Ms. 375/73 enthalten sind (Abb. 3). Die Kurzbeschreibungen informieren in der Regel auch darüber, aus welchem Buch das Fragment ausgelöst wurde.

Zu den übrigen Fragmenten Ms. 375/74 bis Ms. 375/279 finden sich lediglich auf den Schutzmappen rudimentäre Informationen zum Inhalt. Die ungezählten Fragmente (zwei Kisten) befinden sich in Umschlägen, auf denen meist vermerkt ist, aus welchem Band das Fragment ausgelöst wurde (Abb. 4). Für einige der Fragmente sind außerdem auf den Umschlägen Datierungen festgehalten. ${ }^{21}$ Die zwei neuesten gezählten Fragmente - nämlich Ms. 375/278 und Ms. 375/279 - sind Zufallsfunde, die im Jahr 2019 bei einer Sichtung der Fragmentekisten von Jürgen Wolf und der Verfasserin gemacht wurden. Ms. 375/278 wurde von Bernhard Schnell als deutsche Übertragung des ,Macer Floridus', eines mittelalterlichen Standardwerks über Heilkräuter, identifiziert und in das I4. Jahrhundert datiert. ${ }^{22}$ Bemerkenswert ist der Fund dieser makulierten Kräuterheilkunde nicht nur, weil es sich um einen deutschsprachi- 
gen Text handelt, sondern auch, weil medizinische Texte vergleichsweise selten unter Makulaturfragmenten zu finden sind. ${ }^{23}$ Ms. 375/279 ist ebenfalls ein spätmittelalterliches Textfragment, allerdings in französischer Sprache (Abb. 5). Es handelt sich um einen geistlichen Text, der bislang nicht genauer identifiziert werden konnte. Auf dem Fragment ist außerdem der Trägerband vermerkt (C. Peucer, commentar. de praecipuis generibus divinationum $1560.8^{\circ}$ Wittenberg), aus dem es ausgelöst wurde. Beigefügt findet sich in der Mappe außerdem ein Begleitschreiben. ${ }^{24}$

Dass eine systematische Sichtung der Marburger Fragmentekisten eine lohnenswerte und gebotene Aufgabe darstellt und durchaus überraschende Funde zu erwarten sind, zeigen diese jüngsten Neufunde deutscher und französischer Sprache, aber auch das frühe Priscian-Fragment Ms. 375/ra aus der Zeit um 800, das bis 2004 unsigniert und der Forschung damit unbekannt war. Die Pergament- und Papierreste stellen zwar oft nur noch Spuren und Überbleibsel mittelalterlicher Bücher dar, trotzdem handelt es sich um wichtige Quellenmaterialien. Forschungsrelevanz besitzen die Fragmente nicht nur durch ihre schiere Menge, sondern auch wegen der Fülle an Informationen, die sich aus der Zusammenschau selbst kleinster Reste zur frühen Buchproduktion, zur Überlieferungsdichte und der Verbreitung von literarischen Texten und Autoren, aber auch zu Makulierungswellen rekonstruieren lassen.

Gerade neuere Fragmentfunde ${ }^{25}$ haben jüngst wieder gezeigt, dass insbesondere bei den Makulaturmaterialien zwar keine unbekannten Werke gefunden werden, sich dafür aber häufig vergleichsweise frühe Textzeugen von Werken entdecken lassen. Solche Funde sind nicht nur ein quantitativer Zugewinn an Quellenmaterialien, sondern sie ermöglichen der Forschung ein deutlich differenzierteres Bild der Literaturgeschichte. Fragmente, so könnte man sagen, sind ungehobene Datenschätze und so verwundert es kaum, dass das Interesse der Forschung an Fragmenten seit Jahren stetig zunimmt. ${ }^{26}$ Im Kontrast zum wachsenden Forschungsinteresse dieser äußerst forschungsrelevanten und geradezu forschungsbedürftigen Quellen steht allerdings eine dürftige Erschließungslage und Zugänglichkeit. Das ist auch der Grund, warum die Universitätsbibliothek bis zum 5oojährigen Jubiläum der Marburger Philipps-Universität im Jahr 2027 ihre gesamten mittelalterlichen Bestände digitalisieren möchte. Bestandteil dieser Digitalisierungsoffensive wird auch die umfangreiche Fragmente-Sammlung Ms. 375 sein, die bislang nur unzureichende Beachtung fand. Da die Fragmente, nicht katalogisiert und zum Teil noch unbearbeitet sind, stellt das Vorhaben eine Herausforderung dar, denn eine der wichtigsten Voraussetzungen für das Onlinestellen sind hinreichende Erschließungsdaten. Es ist angedacht, für das Erstellen der erforderlichen Metadaten auf die Daten zurückzugreifen, die uns analog in Schriftform auf den Umschlagmappen, im Handschrifteninventar oder in sonstiger Dokumentation vorliegen ${ }^{27}$. Fehlen analoge Erschließungsdaten, sollen die Metadaten anhand leicht $\mathrm{zu}$ bestimmender Merkmale erstellt werden (z.B. Signatur, Sprache, Pergament/Papier). Wichtig ist es, die Angabe der Trägerbände soweit bekannt - in den Metadaten zu erfassen, damit die Herkunft der Fragmente für die Überlieferungsforschung nachvollziehbar bleibt. Die Fragmente und die dazugehörigen Digitalisate sollen im lokalen Bibliothekskatalog verzeichnet und auf dem Publikationsserver der Universitätsbibliothek Marburg als Teil der Digitalen Sammlungen online präsentiert werden. Darüber hinaus können die Daten außerdem zentralen Plattformen wie dem ,Handschriftencensus', dem ,Handschriftenportal', ,Fragmentarium', aber auch der ,Deutschen Digitalen Bibliothek ${ }^{6}$ und der ,Europeana' zur Verfügung gestellt werden. Auf diese Weise wird nicht nur den Forschenden, sondern über den akademischen Bereich hinaus auch der Öffentlichkeit ein bequemer Zugriff und eine Teilhabe an faszinierenden Quellenmaterialien und einzigartigem kulturellen Erbe ermöglicht. 


\section{Kontakt:}

\section{Diana Müller}

Universitätsbibliothek Marburg · Deutschhausstr. 9 35037 Marburg

E-Mail: diana.mueller@ub.uni-marburg.de

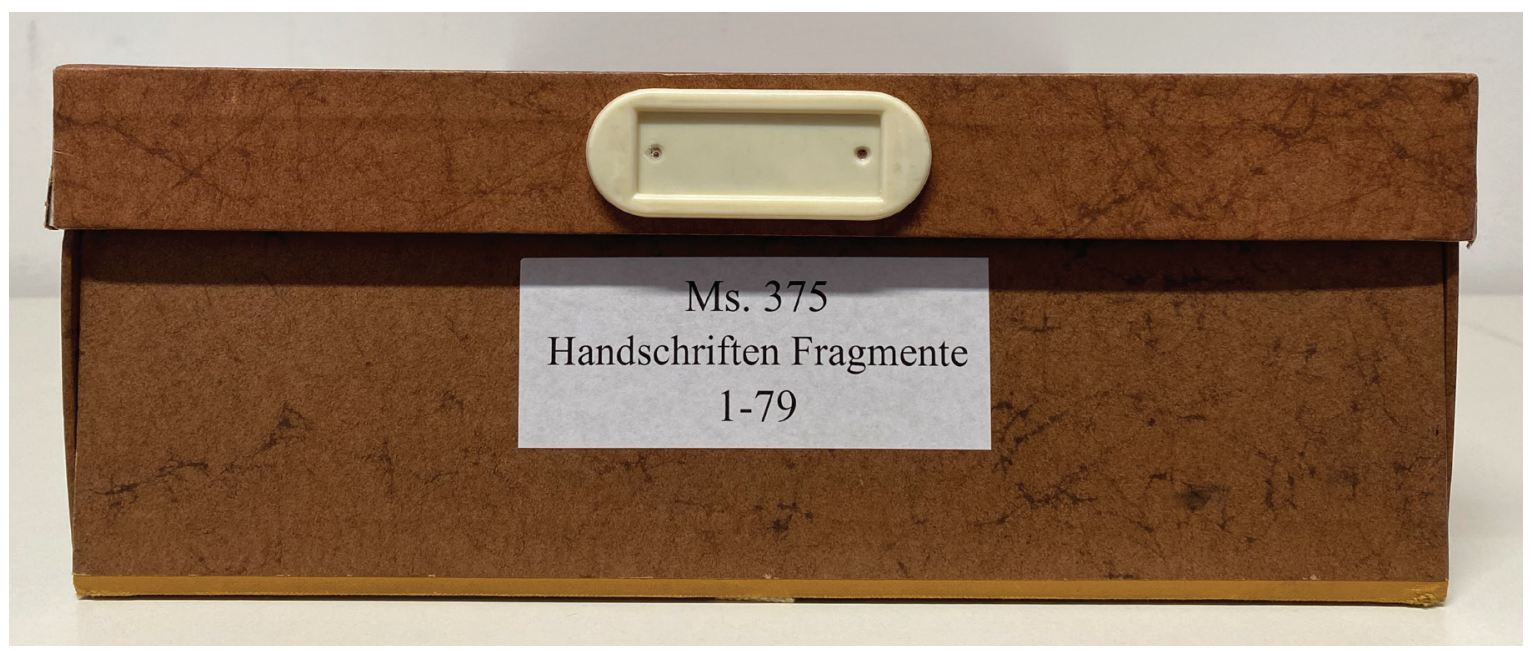

Abb. I: Fragmentekiste Marburg, Universitätsbibliothek, Ms. 375/I-79

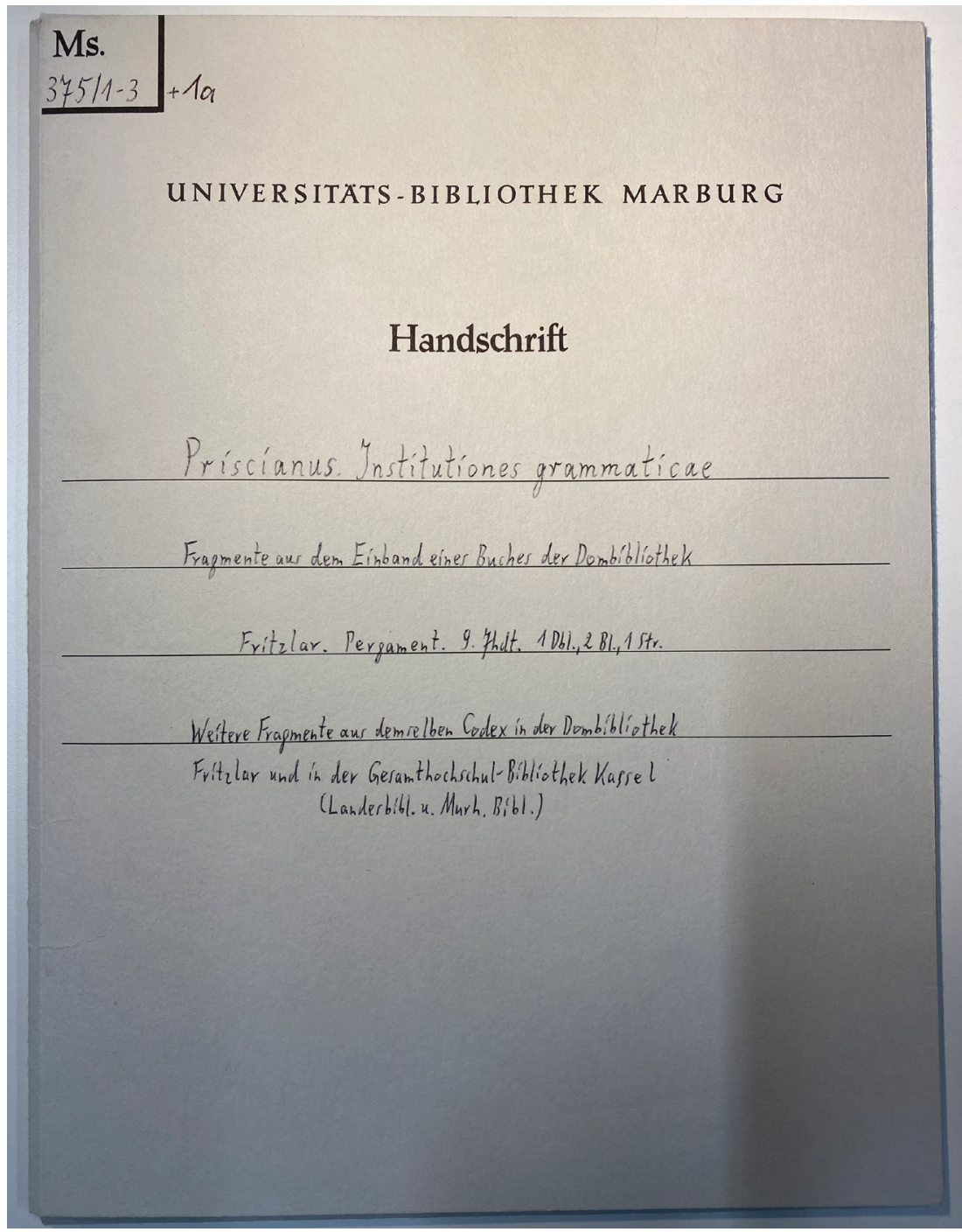

Abb. 2: Marburg, Universitätsbibliothek, Ms. 375/I-3+Ia mit Schutzmappe 


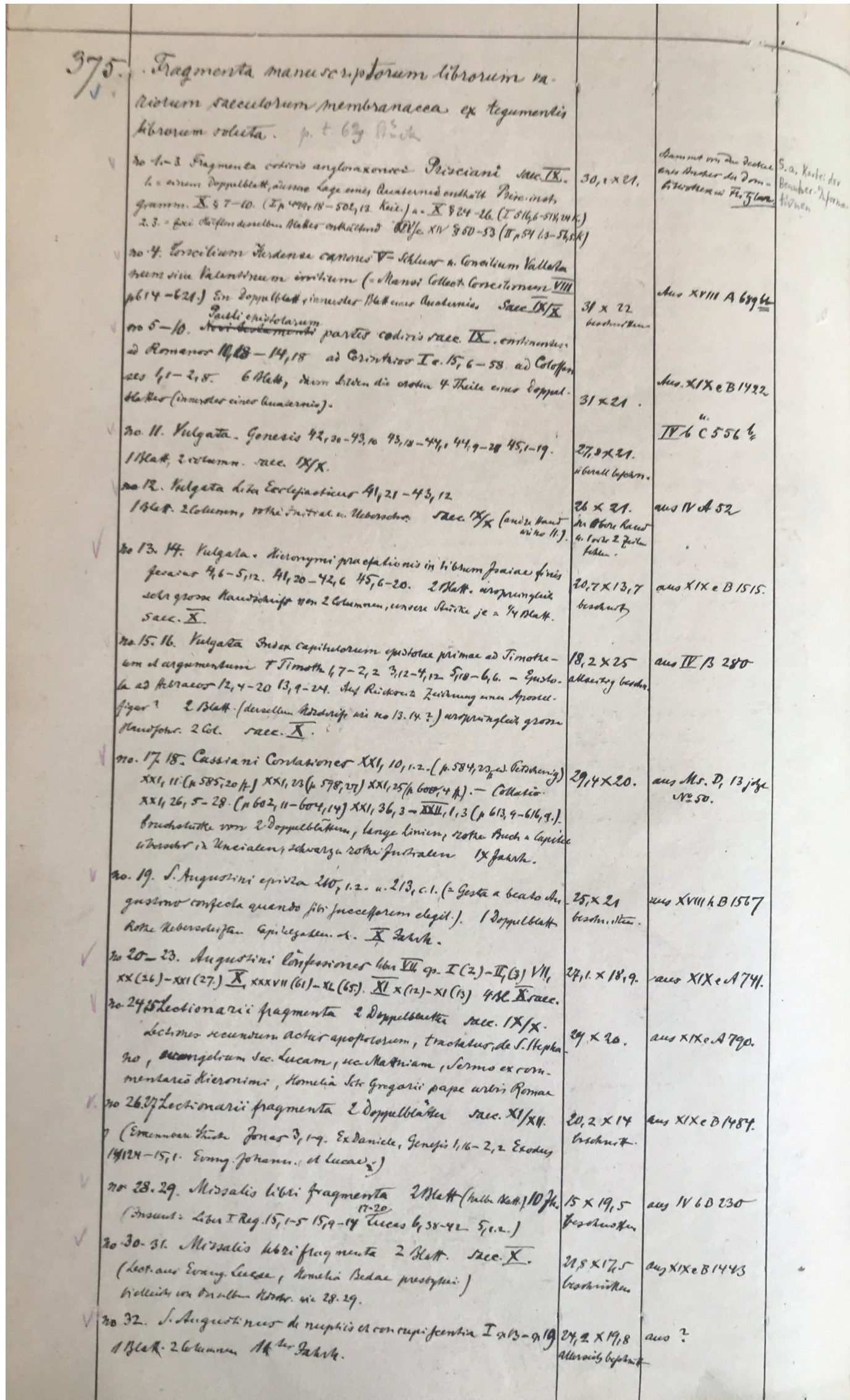

Abb. 3: Marburg, Universitätsbibliothek, Handschrifteninventar (für den Dienstgebrauch).

Eintrag Ms. 375/I-3: Spalte I zeigt die Gruppensignatur Ms. 375, Spalte 2 enthält Informationen zu den einzelnen Fragmenten I-3, Spalte 3 die Maßangaben zu den Fragmenten, Spalte 4 den Hinweis „stammt von dem Deckel eines Buches der Dombibliothek in Fritzlar“, Spalte 5 enthält einen Hinweis von späterer Hand „5.a. Kartei der Benutzer-Informationen“ 


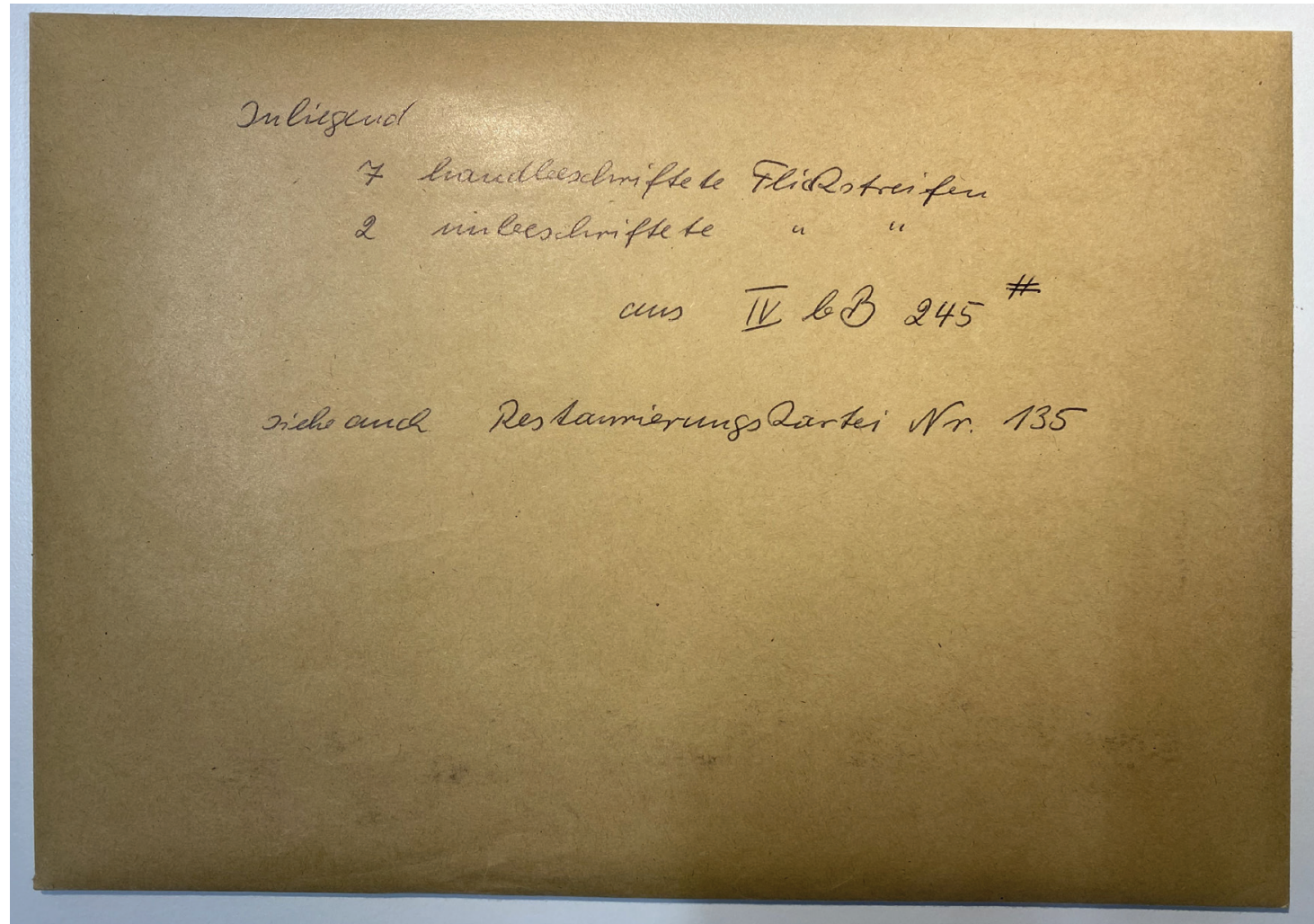

Abb. 4: Marburg, Universitätsbibliothek, Ms. 375/ohne Zählung, Umschlag mit Makulatur

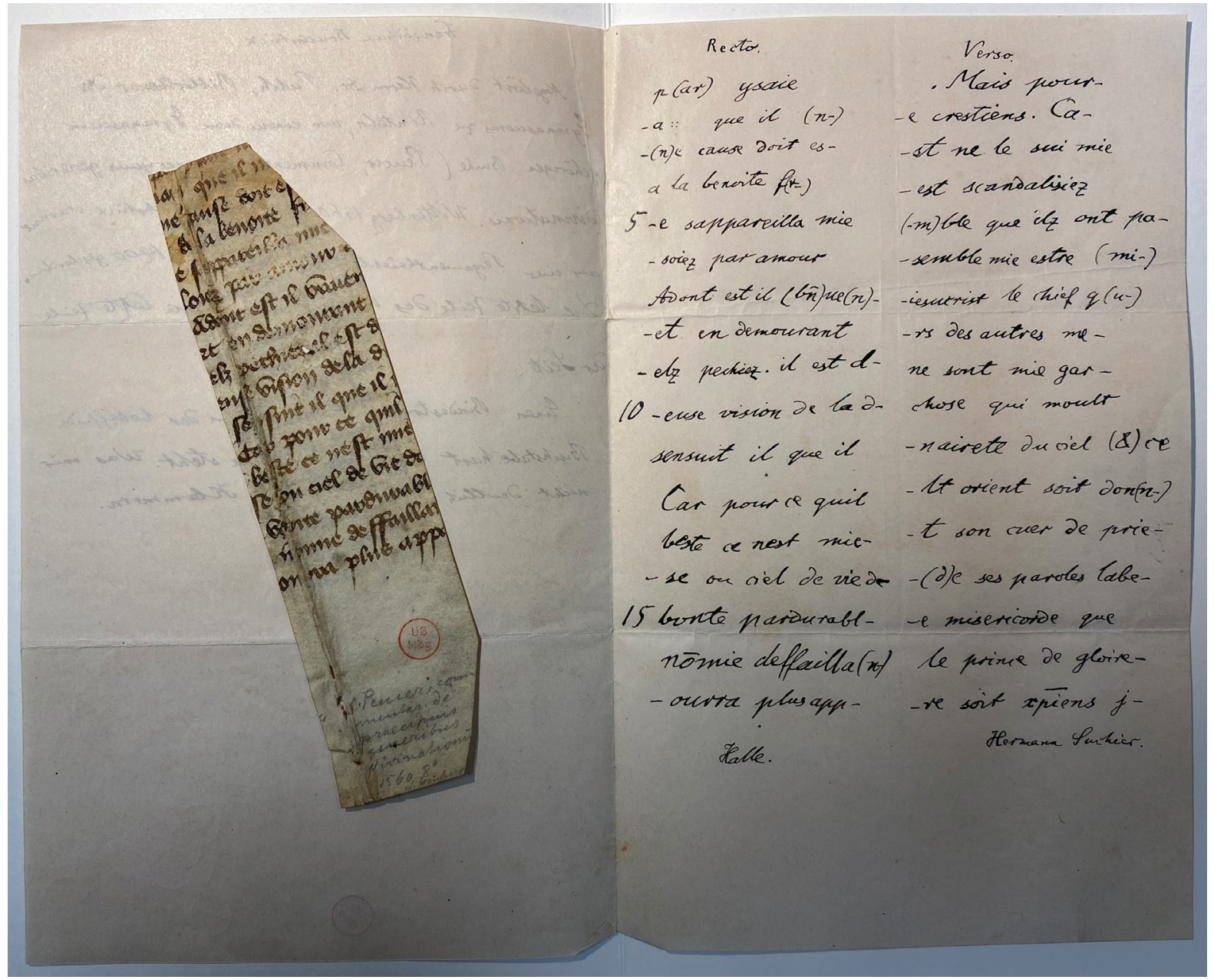

Abb. 5: Marburg, Universitätsbibliothek, Ms. 375/279 mit beigefügter Transkription 


\section{Anmerkungen}

I Sirka Heyne, Die mittelalterlichen Handschriften der Universitätsbibliothek Marburg, Wiesbaden 2002. Die Rezension zum Heyne-Katalog von Martin Schubert in der ZfdA I35 (2006), S. 48I-483, bietet insbesondere eine äußerst nützliche Zusammenstellung der deutschsprachigen Texte und Einträge, die im Register des Heyne-Katalogs nicht gesondert ausgewiesen sind. Der frühere Catalogus codicum manuscriptorum, qui in bibliotheca academia Marburgensi asservantur, Latinorum aus dem Jahr I838 von Carl Friedrich Hermann und der I84I erschienene Nachtragsband mit dem Titel Analecta seien an dieser Stelle der Vollständigkeit halber noch erwähnt.

2 Vgl. Heyne [Anm. I], S. IX und XXVII.

3 Vgl. Heyne [Anm. I], S. IX und XXVII.

4 Vgl. Heyne [Anm. I], S. Xff.

5 Vgl. Heyne [Anm. I], S. XIX.

6 Vgl. Heyne [Anm. I], S. XII.

7 Gundolf Keil, Hans Minner, in: Lexikon des Mittelalters 6 (1993), Sp. 646.

8 Vgl. Heyne [Anm. I], S. XXff.

9 Vgl. Heyne [Anm. I], S. XXIV.

Io Für diesen Hinweis auf die Käufe und Schenkungen bedanke ich mich herzlich bei dem Gutachter bzw. der Gutachterin dieses Beitrags. Ebenso bedanke ich mich für den Hinweis, dass noch immer rund I25 Fragmentblätter und -stücke in den Einbänden der Handschriften stecken - fast alle seien lateinisch, 8o Prozent seien spätmittelalterlich und 40 Prozent seien Liturgica.

II Christian Heitzmann, Aus Alt mach Neu. Fragmente mittelalterlicher Handschriften als Einbandmakulatur in Beständen der Herzog August Bibliothek, in: Biographien des Buches. Hg. von Ulrike Gleixner u.a., Göttingen 2017, S. $367-382$, hier S. 369 .

I2 Dirk Barth, Vorwort, in: Heyne [Anm. I], S. VII.

I3 Gerhard Baader, Hermann, Carl Friedrich, in: Neue Deutsche Biographie 8 (1969), S. 66ı. Franz Gundlach, Catalogus professorum academiae Marburgensis I527-I9I0, Marburg 1927, S. 336f.

I4 Vgl. insbesondere den Abschnitt „History of the fragment collection at ULB“ in: Ivana Dobcheva, Christoph Mackert, Manuscript Fragments in the University Library, Leipzig. Types and Cataloguing Patterns, in: Fragmentology I (2018), S. 83-IIo, hier S. 84-86 [DOI: I0.24446/rx89].

Is Für diese und zahlreiche weitere Informationen zur Marburger Fragmente-Sammlung bedanke ich mich beim Leiter der Abteilung Historische Bestände der Universitätsbibliothek Marburg, Bernd Reifenberg.

I6 Vgl. Heyne [Anm. I], S. XXVII.

17 Die Fragmente in Ms. 375 haben ungefähr zur Hälfte eine Stückzählung, der Rest ist noch ungezählt. Alle Fragmente befinden sich innerhalb der Kisten in eigenen Schutzmappen bzw. Schutzumschlägen.

I8 Vgl. Heyne [Anm. I].

I9 Nach Auskunft von Bernd Reifenberg (UB Marburg) kam der Hinweis auf den Pergamentstreifen im Jahr 2004 schriftlich von Elke Krotz: Ihr sei aufgefallen, dass sich auf dem schmalen Streifen Reste eines Textes befinden, der in den Handschriftenkatalogen nicht erfasst und der einzige Rest eines eigenen Blattes sei.

2o Bernhard Bischoff, Katalog der festländischen Handschriften des neunten Jahrhunderts (mit Ausnahme der wisigotischen). Teil II: Laon-Paderborn. Aus dem Nachlass hg. von Birgit Ebersperger, Wiesbaden 2004.

2I Die Datierungen stammen nach Auskunft von Bernd Reifenberg von Uwe Bredehorn, dem Leiter der Historischen Sammlung bis 2007. Einer der Vorgänger hat die Erforschung dieses Bestands bis Ms. 375/73 vorangetrieben und im Inventar festgehalten. Uwe Bredehorn hat die Beschreibungen fortgesetzt bis Ms. 375/277.

22 Eine Publikation zum Marburger ,Macer'-Fragment wird von Bernhard Schnell vorbereitet. 
23 Claudia Sojer, Walter Neuhauser, Manuscript Fragments in the University Provincial Library of Tyrol at Innsbruck, in: Fragmentology II (2019), S. I4I-I63, hier S. I58 [DOI: I0.24446/ia4e].

24 Das undatierte Begleitschreiben stammt von Paul Pulch und lautet: „Französisches Bruchstück. Abgelöst durch Herrn Dr. Pulch, Bibliothekar des Gymnasiums zu Rinteln von einem dem Gymnasium gehörigen Bande (Peucer, Comment. de praecipius generibus divinationis. Wittenberg $1560.8^{\circ}$ ). Das Bruchstück stammt aus einer Pergamenthandschrift und ist um I400 geschrieben. Die letzte Zeile des Bruchstücks war die letzte Zeile der Seite. Einen Bindestrich setze ich, wenn der betreffende Buchstabe hart an der Schnittlinie steht. Was mir nicht deutlich ist, setze ich in Klammern.“

25 ,Der Rosendorn' (Melk, Stiftsbibliothek, Fragm. germ. 8, um I300), ,Macer', dt. (Marburg, Universitätsbibliothek, Ms. 375/278, I4. Jh.). Zum Fragmentfund mit dem bislang frühesten Beleg des ,Parzival‘ Wolframs von Eschenbach: Matthias Eifler, Christoph Mackert, Michael Stolz, Leipziger Handschriftenfunde I. Ein neu aufgefundenes Fragment von Wolframs ,Parzival' aus Naumburg, in: ZfdA I43 (20I4), S. 306-332. Herausragend ist außerdem der Fund eines Doppelblatts aus dem ersten Viertel des des 8. Jahrhunderts mit althochdeutschen Glossen aus dem späten 8. Jahrhundert in der Fragmente-Sammlung der Universitätsbibliothek Leipzig: Christoph Mackert, Hans Ulrich Schmid, Ein spätmerowingisches Handschriftenfragment mit frühen althochdeutschen Glossen. Zum fragmentum latinum 430 der UB Leipzig, in: athe in palice, athe in anderu sumeuuelicheru stedi. Raum und Sprache. Festschrift für Elvira Glaser zum 65. Geburtstag, hg. von Andreas Nievergelt und Ludwig Rübekeil, Heidelberg 2019, S. I43-г6r.

26 Fragmentarium. Laboratory for Medieval Manuscript Fragments (Universität Fribourg): <https://fragmentarium. ms/> (I2.6.2020); Fragmentology. A Journal for the Study of Medieval Manuscript Fragments: <http://fragmentology.ms/> (I2.6.2020); Fragmente. Der Umgang mit lückenhafter Quellenüberlieferung in der Mittelalterforschung, hg. von Christian Gastgeber u.a., Wien 2oı०; Fragment und Makulatur. Überlieferungsstörungen und Forschungsbedarf bei Kulturgut in Archiven und Bibliotheken, hg. von Hanns Peter Neuheuser und Wolfgang Schmitz, Wiesbaden 2015; Das Ganze im Fragment. Handschriftenfragmente aus kirchlichen Bibliotheken, Archiven und Museen, hg. von Alessandra Sorbello Straub, Petersberg 20I5; Das Ganze im Fragment. Zerstörte und wiederentdeckte Schätze aus kirchlichen Bibliotheken, Archiven und Museen. Beiträge der 2. Tagung der gemeinsamen Altbestandskommission von AKThB und VkwB, hg. von Alessandra Sorbello Staub, Regensburg 2018 (Jahrbuch kirchliches Buch- und Bibliothekswesen N.F. 4,20I7).

27 Ms. 375/I bis Ms. 375/73: Kurzbeschreibungen im Handschrifteninventar (vgl. dazu Abb. 3) und Erschließungsdaten auf den Schutzmappen (vgl. dazu Abb. 2), Ms. 375/74 bis Ms. 375/279: Erschließungsdaten auf den Schutzmappen (vgl. dazu Abb. 2), Ms. 375/ [2 Kisten ungezählt]: Informationen zu den Trägerbänden auf den Umschlägen (vgl. dazu Abb. 4), zum Teil auch Datierungen. Neben diesen unveröffentlichten und bisher nur für den Dienstgebrauch zugänglichen Daten finden sich Beschreibungen im Katalog von Heyne [Anm. I] zu Ms. 375/I-3, Ms. 375/17-I8, Ms. 375/28-3I und Ms. 375/85-86 sowie im Katalog von Bischoff [Anm. 20] zu Ms. 375/I-3, Ms. 375/5-10, Ms. 375/II, Ms. 375/I2, Ms. 375/I3-I6, Ms. 375/I7-I8, Ms. 375/19, Ms. 375/24-25, Ms. 375/69-70 und Ms. 375/74. 